$36 \mid 2006$

De quelques enjeux et usages historiques du Français fondamental

\title{
Français élémentaire, débats publics et représentations de la langue
}

Daniel Coste

\section{(2) OpenEdition}

\section{Journals}

Édition électronique

URL : https://journals.openedition.org/dhfles/1181

DOI : $10.4000 /$ dhfles. 1181

ISSN : 2221-4038

Éditeur

Société Internationale pour l'Histoire du Français Langue Étrangère ou Seconde

Édition imprimée

Date de publication : 1 janvier 2006

Pagination : 13-33

ISSN : 0992-7654

Référence électronique

Daniel Coste, «Français élémentaire, débats publics et représentations de la langue », Documents pour I'histoire du français langue étrangère ou seconde [En ligne], 36 | 2006, mis en ligne le 24 août 2011, consulté le 27 mai 2021. URL : http://journals.openedition.org/dhfles/1181 ; DOI : https://doi.org/ $10.4000 /$ dhfles. 1181

Ce document a été généré automatiquement le 27 mai 2021.

(c) SIHFLES 


\title{
Français élémentaire, débats publics et représentations de la langue
}

\author{
Daniel Coste
}

1 Un jour d'octobre 2004, dans le journal Le Monde, grâce à la rubrique « Il y a cinquante ans dans Le Monde ", un article de Bertrand Poirot-Delpech publié en 1954 se trouvait reproduit, qui annonçait alors la publication du petit fascicule Le Français élémentaire, produit d'une enquête linguistique qui avait duré environ trois ans et suscité de vives polémiques.

2 Il vaut la peine de revenir sur cet épisode qui intéresse les politiques de la langue, l'histoire de l'enseignement du français langue étrangère ou seconde, et aussi, largement, les sciences du langage. L'élaboration du Français élémentaire soulève en effet des questions d'ordre historique. Questions qui touchent notamment aux relations entre corpus linguistiques, étude et représentation de l'oral, détermination des contenus d'enseignement.

3 Histoire courte certes, relevant du domaine de l'histoire contemporaine, où l'épisode de l'élaboration de ce qui fut vite rebaptisé Français fondamentalse présente comme un point nodal, comme un moment particulièrement significatif où apparaissent soit en pleine lumière, soit plus dissimulées, les tensions qui marquent, d'une part, ces jeux entre corpus, oralité, interventions dans le domaine éducatif ou dans l'aménagement linguistique, d'autre part les représentations sociales auxquelles ces rapports donnent lieu, y compris, bien entendu, les représentations que proposent, en un temps et dans un contexte donnés, les modèles ou les conceptions scientifiques.

4 Mais le propos ici est modeste : une lecture, une relecture de certains aspects et de certains moments de ce qu'on peut appeler l'aventure du Français fondamental. Relecture nécessairement partielle et sélective de l'abondante production discursive qu'a suscité à l'époque le projet, mais lecture directe aussi, recourant à de nombreuses citations d'articles et de coupures de presse, de manière à donner à voir, dans leur formulation même, des textes dont la vigueur polémique peut aujourd'hui surprendre. Dans ce même recueil, P. Rivenc, acteur majeur de la réalisation de l'enquête et de bien des développements qui suivirent, apporte un témoignage tout aussi direct sur le 
rapport à l'oral qu'entretenaient certains protagonistes de l'élaboration du Français fondamental. Et J. C. Chevalier traite d'une des pièces importantes du dossier : le petit opuscule intitulé Français élémentaire, non, publié par M. Cohen «et un groupe de linguistes " après la sortie de la brochure Français élémentaire. En conséquence, les aspects développés par ces acteurs / auteurs ne seront pas ici abordés en détail.

Deux précisions quant aux sources et à la désignation :

6 - Les archives relatives à l'élaboration du Français élémentaire sont très réduites aujourd'hui, du moins à l'École normale supérieure Lettres et Sciences humaines, à Lyon. Déménagements successifs et absence de précautions particulières ont eu raison de bien des documents. On doit ce qui demeure à la vigilance de M. T. Moget et de $\mathrm{H}$. Besse.

7 - Le titre du colloque de décembre 2005 est quelque peu anachronique, dans la mesure où l'appellation Français fondamentaln'apparaît qu'à la fin des années 1950, le fascicule paru en 1954 portant l'intitulé Français élémentaireet certaines des débats du tout début des années 50 faisant état d'un «français de base » que, dans une visée polémique, d'aucuns auront tôt fait de rebaptiser «basic French », en référence allusive à la bien différente entreprise qu'avait pu être le Basic English.

Quant au parcours ici proposé, il partira des origines du projet et des circonstances historiques où il s'inscrit, pour ensuite aborder les aspects techniques et linguistiques des débats qui ont accompagné le processus et marquer les enjeux de politique linguistique et de politique "tout court» qui donnent lieu à l'époque à vives controverses. Une conclusion permettra d'interroger le rôle de révélateur qu'a pu avoir l'élaboration du Français élémentaire.

\section{Origines du projet et circonstances historiques}

\subsection{Une décision fondatrice et un contexte complexe}

C'est en 1947 qu'une recommandation de l'UNESCO souligne l'importance, d'une part, de la diffusion des grandes langues de communication internationale (essentiellement l'anglais et le français), d'autre part, de la promotion des langues non pleinement grammatisées, telles bon nombre des langues africaines alors. Le linguiste A. Sauvageot, professeur à l'École des langues orientales, présente le dossier au Comité de l'Éducation de base de la Commission française pour l'UNESCO et le dossier fait son chemin : il y a eu un Basic English, on aura, pour les mêmes raisons de diffusion et d'alphabétisation, mais suivant des principes tout différents, un français de base. Le piquant de l'affaire, rétrospectivement, est que le projet est présenté par Le Monde, par la plume de Bertrand Poirot-Delpech ${ }^{1}$, sous l'espèce d'un partage de zones d'influence qui peut aujourd'hui sembler pour le moins brutal.

Le ministre de l'éducation nationale, à la demande de M. Abraham, directeur du service universitaire des relations avec l'étranger, vient d'ouvrir un crédit de 3 millions et demi de francs pour préparer, à l'exemple du "basic english ", une langue française de base, assimilable rapidement par les populations les moins perméables à notre culture. [...]

L'idée d'une langue française simplifiée remonte à 1947. L'U.N.E.S.C.O., qui a posé comme principe que le niveau de vie des hommes dépend de leur instruction, convoquait à cette époque des experts linguistes de nombreux pays afin de créer des langues dites de "base ", capable de diffuser rapidement dans les pays les 
« moins évolués » les notions les plus simples d'hygiène, de justice, de morale et de culture.

A. Sauvageot, qui avait été invité par l'U.N.E.S.C.O. à cette conférence, obtint que le français fût adopté en Europe et dans la majeure partie de l'Afrique. Il serait également admis en concurrence en Amérique du sud, dans le Moyen et l'Extrême Orient. Partout ailleurs, l'anglais prévaudrait. [...]

En entreprenant leurs recherches pour créer un français de "base », les spécialistes ne pensent pas nuire à l'effort que la France poursuit dans les territoires d'OutreMer pour développer sa langue et sa culture, mais au contraire l'appuyer.

Le français de «base » doit permettre un dialogue, même rudimentaire, avec tous. Son rendement doit être immédiat. Si nous ne préparons pas ce dialogue, d'autres l'établiront à notre place. Les méthodes modernes d'imprégnation linguistique rendent en effet la concurrence de plus en plus menaçante.

Une première méthode pouvait tenter les spécialistes: former une langue de «base» en partant d'une sélection empirique des mots les plus courants. Elle a le mérite d'être expéditive. Les Américains ont rédigé aussi un dictionnaire de «fréquence » des formes françaises les plus usitées, mais il n'est guère utilisable pour la conversation banale, puisqu'il a été établi d'après des textes littéraires.

Pour éviter cette fausse manœuvre, les experts français calculeront plutôt la fréquence des mots dans des dialogues courants enregistrés sur disques : dialogues d'hommes d'affaires, d'ouvriers étrangers, etc.

Ces documents seront ensuite interprétés par les spécialistes, qui élaboreront une " grammaire parlée » et dresseront la liste d'un français qui comportera environ un millier de mots. [...] Le Monde, $1^{\mathrm{er}}$ novembre 1951)

10 Article remarquable en cela que beaucoup de ce qui suivra dans le débat public s'y trouve en germe: la notion de «français de base ", la référence à l'UNESCO et à l'éducation de base, le rappel de Basic English et la différence méthodologique majeure que constituera le recueil d'un corpus oral de conversations. Ajoutons que bien des formulations du futur académicien paraissent ici empreintes des représentations et des désignations de l'époque.

11 Nous sommes au début des années 50, période marquée par l'instabilité des gouvernements de la Quatrième République, mais aussi par le développement économique, par la Guerre froide, mais aussi par une guerre moins froide en Indochine, par l'Alliance atlantique et par des campagnes anti-américaines d'un Parti communiste "pesant» près de $25 \%$ des voix dans les grandes élections, par la mise en place européenne d'une CECA (Communauté économique du charbon et de l'acier) et par un projet d'armée européenne qui échouera à naître. Période marquée par l'évolution des politiques coloniales : territoires et non plus colonies d'une France d'Outre-Mer ${ }^{2}$ sur l'avenir de laquelle on s'interroge.

12 Dans cet environnement complexe, envisager un renforcement de l'éducation de base pour l'espace colonial, c'est certes faire œuvre de «civilisation », mais aussi, pour beaucoup, d'intérêt bien compris pour la nation. L'extension du français par l'instruction et l'alphabétisation peu servir la France, quoi que réserve l'avenir.

\subsection{Ministères et ENS}

13 C'est toute fois le Ministère de l'Éducation qui s'engage dans l'entreprise de l'élaboration d'un français de base, les services de la France d'Outre-Mer se montrant nettement plus réservés, pour ne pas dire défavorables. Au demeurant, l'avenir verra le 
succès du Français élémentaire ailleurs que dans cette France d'Outre-Mer à laquelle il était d'abord destiné.

Les pouvoirs publics pressentent l'affaire comme délicate et estiment qu'il convient d'aller vite. Un "centre d'étude du Français élémentaire » est créé et installé à l'École normale supérieure de Saint-Cloud, avec financement par le biais de la Commission française pour l'UNESCO. L'ENS n'est pas l'université et ne se caractérise pas par une forte tradition de recherche (mais quelle université a alors une telle tradition dans le domaine linguistique, et où trouve-t-on autre chose qu'une philologie tenant à des individualités, non à des laboratoires ?). L'ENS de Saint-Cloud se situe alors sur une trajectoire ascendante : longtemps cantonnée à la formation de cadres pour le primaire et le primaire supérieur, elle a désormais, comme Ulm, et Sèvres, une fonction de préparation aux agrégations. Elle met en place des centres pédagogiques, dont le premier sera le Centre audio-visuel, et elle entretient depuis longtemps, par nombre de ses anciens élèves et par l'engagement personnel de certains de ses cadres, des rapports avec l'éducation à l'étranger et singulièrement dans l'empire colonial ${ }^{3}$. En bref, on a là une institution de notoriété suffisante et de bon statut, dont l'égide est plausible pour héberger la petite équipe chargée de l'enquête.

\subsection{Garants scientifiques, directeur et maître d'œuvre}

Quels hommes pour la mener? A. Sauvageot a été la cheville ouvrière première. Ancien de l'ENS de la rue d'Ulm, professeur à l'Ecole des langues orientales, spécialiste de finno-ougrien, il a fait une partie de sa carrière à l'étranger. Responsable d'un Centre de recherche pour les langues d'Outre-Mer créé comme pendant du Centre d'étude du Français élémentaire ${ }^{4}$. A. Sauvageot s'intéresse à l'oralité et publiera sur cet objet des travaux originaux pour l'époque.

Mais il faut un spécialiste de français pour assurer la responsabilité scientifique du projet. C'est G. Gougenheim qui est pressenti et qui accepte cette tâche. Lui aussi ancien d'Ulm, seiziémiste, grammairien et lexicologue dont la thèse complémentaire a porté sur la langue populaire dans le premier quart du XIXe siècle, ce disciple de F. Brunot, d'E. Huguet et $\mathrm{E}$ Gilliéron est alors âgé d'une cinquantaine d'années et enseigne à la Faculté des Lettres de Strasbourg. Il a travaillé sur le système phonologique et publié dès avant 1940 une grammaire structurale du français. Chevalier et Encrevé (2006) voient en lui un « isolé ", auquel la compétence, le sérieux et la curiosité scientifique n'ont pas suffi à conférer un prestige et un poids comparables à ceux d'autres francistes, tels R. L. Wagner, C. Bruneau ou A. Dauzat.

Sous la direction de Gougenheim, la cheville ouvrière et l'animateur infatigable du Centre d'études est P. Rivenc, jeune ancien de Saint-Cloud, spécialiste de lettres modernes, professeur à l'Ecole normale d'Evreux quand on vient le chercher pour le projet d'élaboration d'un Français élémentaire. Militant de l'éducation de base, actif dans Peuple et Culture, Rivenc sera, à moins de trente ans, le maître d'œuvre de l'entreprise et, une fois réalisée l'enquête, engagera le Centre d'étude dans des activités de recherche, de création pédagogique et d'innovation méthodologique qui conduisent, en 1959, à la création du CREDIF. 


\subsection{Une commission de suivi du projet}

Une commission est mise en place pour accompagner le projet. L'équipe du Centre doit lui présenter rapports et résultats et c'est cette commission qui assurera la mise en forme finale des listes retenues pour constituer le Français élémentaire, une fois réalisées les enquêtes lexicales de fréquence et de disponibilité. Font partie de cette commission, entre autres personnalités, des linguistes et philologues tels qu'E. Benveniste, M. Lejeune, F. Mossé. R. L. Wagner, nommé dans la commission, n'y participera pas et A. Dauzat refuse d'en être membre.

Cet organe est loin d'avoir eu à l'époque une fonction simplement symbolique. C'est ainsi que Benveniste joue un rôle important dans les choix touchant à la grammaire. C'est aussi la commission qui expurgera la liste finale d'un certain nombre de mots «familiers » que leur fréquence aurait dû faire retenir et qui, pour nombre d'entre eux, constituaient des marques de l'oralité (voir Gadet, à paraître).

\section{Les débats d'ordre technique et méthodologique}

A. Dauzat, dont on vient de noter qu'il avait refusé de faire partie de la Commission créée par le ministre de l'Éducation nationale, est un des principaux protagonistes du débat linguistique technique à propos de l'élaboration du Français élémentaire. Franciste moderniste, promoteur et défenseur de l'agrégation de lettres modernes (qui fut un lieu d'âpres combats), c'est l'animateur majeur de la grande revue de référence, Le Français moderne. Présent dans les journaux, il publie notamment des articles dans Le Monde. Forte personnalité, mais d'une autre génération que les protagonistes du Français élémentaire (il est âgé de plus de 70 ans à l'époque), il est résolument favorable à une simplification des contenus d'enseignement en vue d'une éducation de base. Mais il s'interroge sur différents aspects de l'enquête entreprise. Selon trois axes de questionnement :

\subsection{A-t-on besoin d'une enquête lourde?}

La base commune à tout français (comprenant les verbes, les noms abstraits essentiels, les adjectifs courants, les noms de parties du corps, de parenté, etc.) peut être obtenue facilement par le dépouillement d'un petit Larousse, confié - pour éviter les choix subjectifs - à trois ou quatre personnes différentes, appartenant à divers degrés de l'enseignement et qui devraient éliminer tout ce qui est au-dessus de la capacité des débutants : les résultats seraient ensuite confrontés. (Le Monde, 13 mai 1952).

Le recours au dictionnaire, le choix des dépouillements, autant de traits qui trahissent la représentation très scolaire que Dauzat propose de ce français pour l'éducation de base, là où les promoteurs du Français élémentaire entendent recueillir des « usages oraux authentiques ». Mais les critiques de Dauzat vont plus loin et apportant de l'eau au moulin des opposants au projet...

\subsection{Peut-on se satisfaire d'une enquête faite en France?}

Un vocabulaire doit être adapté aux besoins de ceux qui l'apprennent, besoins qui varient considérablement dès qu'on s'éloigne de la métropole. Une enquête de fréquence à Paris était inutile. [...] Comme l'ont judicieusement fait observer des 
représentants qualifiés du ministère de la France d'Outre-Mer, ce n'est pas à Paris que l'enquête doit être faite, mais à Alger, à Dakar, à Brazzaville, à Tananarive, à Saigon. première applique au français le qualificatif de « langue étrangère » pour le contexte de l'Outre-Mer colonial, caractérisation plutôt hardie à l'époque. La seconde permet au linguiste de mettre en cause les carences de la formation en linguistique des enseignants et de leurs responsables ${ }^{6}$. Mais si $\mathrm{M}$. Cohen tire ici plusieurs lièvres à la fois, il n'implique pas que l'enquête serait en tous points inutile. Lui-même s'intéresse à ce qu'on appellerait aujourd'hui de grands corpus, croit à l'importance des enquêtes linguistiques ${ }^{7}$ et, à la différence de Dauzat, ne s'en prend pas de front aux choix 
méthodologiques opérés pour le recueil des données sonores. Ses critiques linguistiques - vives - seront d'une autre nature (voir ici même l'analyse que fait J. C. Chevalier de l'opuscule Français élémentaire? Non, publié après la sortie du fascicule du Français élémentaire).

Au moment où s'engage l'enquête, c'est sur le terrain politique que la polémique la plus violente va se manifester et $\mathrm{M}$. Cohen en sera un des acteurs principaux. Polémique touchant à la politique de la langue certes, mais aussi, d'un bord et de l'autre, à la politique « tout court».

\section{Les mises en cause politiques et idéologiques}

C'est l'article de B. Poirot-Delpech, cité plus haut, qui a mis le feu aux poudres, avec ses accents de Realpolitik (du style: si on n'y va pas, d'autres le feront) et de Yalta linguistique (l'anglais et le français se partageant le monde). Et, dans le contexte historique des années 1950, les réactions politiques ne vont pas manquer, à gauche comme à droite, faisant passer au second plan les aspects méthodologiques et linguistiques. Différents angles de mise en cause du projet peuvent être distingués : la bonne utilisation des financements, les intentions cachées de l'entreprise, les risques de mutilation de la langue.

\subsection{Le financement de la recherche}

Dans son article dans Lettres françaises du 7 février $1952^{\circ}$, M. Cohen développe en premier lieu un commentaire sur le financement du projet. Commentaire qui vaut d'être cité, car il montre bien l'intérêt du linguiste pour la constitution d'atlas et de corpus en vue de l'étude du français et de la variation :

Jusqu'à présent l'étude du français avait vécu sous le régime de la restriction ou de l'éteignoir. L'atlas linguistique de la France de Gilliéron-Edmont, en 1900, fait avec une subvention infirme; atlas particuliers de diverses régions, sans subvention; nouvel atlas mis en train en 1939, pas de subside. Inventaire de la langue française, c'est-à-dire mise en chantier du grand dictionnaire de toutes époques jusqu'à nos jours qui nous manque, crédits ridiculement insuffisants, depuis 1936. Constitution d'une phonothèque des parlers locaux et des grandes voix de France, inaugurée par entreprise privée, à peine continuée [...]

Aujourd'hui, changement de décor. Des crédits, on en a trouvé (oh! n'ayez pas peur, des crédits modestes, sur le module instruction publique, pas au taux militaire) [...] Comment cet événement s'est-il produit?

Ce n'est pas un revirement spontané. Ce sont les échos d'une grande opération atlantique, avec action dans l'UNESCO et à côté de l'Unesco. Affaire complexe, qui ne se prête pas facilement à un résumé. Aussi bien, il serait erroné de méconnaître, à côté des impérialismes plus ou moins avoués, des bonnes intentions détournées de leur but et même des technicités mal conscientes des fâcheux aboutissants de leurs travaux. (Les Lettre françaises, 7 février 1952) ${ }^{9}$.

On notera que, si Cohen incrimine les pouvoirs publics, accusés de ne pas financer les recherches sur le français, il ménage ici les protagonistes scientifiques de l'enquête du Français élémentaire (Sauvageot et Gougenheim), surtout taxés de naïveté et de se laisser embarquer dans une entreprise « atlantique». 
argumentation est limpide : " il y a des crédits pour ce projet, alors que d'autres n'en ont pas obtenu ou en ont cruellement manqué, c'est que le 'basic french' répond à une intention politique. Le débat est alors à engager sur un autre plan.

\subsection{Des visées politiques à dénoncer?}

L'article des Lettres françaisesse poursuit sans ambiguïté :

Le cadre général qu'il nous fait connaître, c'est, sur le terrain linguistique comme sur les autres, l'offensive américaine, avec une apparence hypocrite de défensive du côté de l'Atlantique.

et M. Cohen met en avant différentes affirmations quant aux visées inavouées qu'il prête au projet: perspectives militaires (lien ave le projet d'armée européenne) ou civile (tant en contexte colonial qu'ailleurs). Son analyse, informée et détaillée autant qu'orientée, apparaît aujourd'hui biaisée par le propos politique, mais bien moins virulente que d'autres prises de position dans la presse. On en retiendra ici, dans ces mêmes Lettres françaises, et peu après l'annonce signée de Poirot-Delpech dans Le Monde (auquel il se réfère directement, notamment à un paragraphe où il était fait mention de l'emploi du Basic English par l'armée britannique dans la formation de troupes hindoues), un bref article intitulé « Il y a culture...et Kultur ! » :

[...] L'aveu est clair ! Il s'agit de créer une langue destinée à faciliter la préparation de la « croisade » occidentale, langue créée, comme le prouve l'exemple cité par Le Monde à l'usage des réserves coloniales de chair à canon que l'on essaiera, une fois de plus, de lancer en avant pour épargner les «races supérieures (Les Lettres françaises, 7 novembre 1951)

Dans L'Humanité du 1er janvier 1952, quotidien du Parti communiste, D. Lazard, sous le titre « Complot contre la langue française » pousse le bouchon encore plus loin et écrit notamment :

Il s'agit donc uniquement de faire de la langue française, réduite à sa plus simple expression, et où le vocabulaire militaire jouera le rôle principal, un instrument de la guerre préparée par les impérialistes américains. Les Français doivent constituer en Afrique les cadres subalternes de l'armée atlantique, et leur langue, réduite à un moignon servir à recruter et à instruire la chair à canon fournie par les peuples d'Afrique. Ici comme ailleurs, c'est l'action du peuple français, alliée à celle des peuples colonisés, qui fera échec à ces criminels desseins. (L'Humanité, $1^{\mathrm{er}}$ janvier 1952)

Nul besoin de souligner combien ces attaques, lancées avant même que l'enquête linguistique soit pleinement engagée, ont pu affecter des hommes comme Gougenheim, Sauvageot, Rivenc, même si ces derniers se firent un principe de ne pas se placer sur ce terrain ${ }^{10}$ et ne ripostèrent qu'après 1954, une fois publié Le Français élémentaire (voir notamment Gougenheim 1956, Rivenc 1956), la résistance au projet étant alors toujours vive, comme en témoigne la parution de Français élémentaire? Non (voir ici même la contribution de J. C. Chevalier) et comme en atteste aussi le quasi autodafé préconisé par le groupe communiste de l'Assemblée nationale, à l'occasion d'une proposition de loi (qui ne verra jamais le jour) :

Les membres du groupe communiste à l'Assemblée nationale [...] ont déposé une proposition de loi tendant à réaliser la réforme de l'enseignement, qui a été publiée en annexe au procès verbale de la séance du 10 mars 1955 [...] L'article 16, à trois alinéas, porte au deuxième: il est interdit de mettre en pratique à un degré quelconque de l'enseignement et sur une portion quelconque du territoire soumis à 
l'autorité française, un système quelconque comportant l'appauvrissement et la mutilation de la langue française (grammaire et vocabulaire), spécialement le système connu sous le nom de Français élémentaire ('basic french') » (in : Français élémentaire? Non, 110-111) française », c'est une autre ligne critique qui se trouve tracée, et cela aussi depuis la première annonce du projet. Et sur cette ligne, d'autres que les linguistes et journalistes communistes ne manquent pas de se positionner.

\subsection{Une attente à l'intégrité de la langue française ?}

L'article déjà cité des Lettres françaises, dont le titre joue, de manière fort peu élégante, sur culture et Kultur, affirme, à propos du français de base : « Une telle langue ne saurait en aucun cas porter le message d'une culture. Lire Rabelais ou Descartes, Heine ou Shakespeare avec huit cents mots! Laissez-nous rire!»; Et, sous des formes diverses, l'argument est utilisé à droite comme à gauche. Les académiciens français tels que $\mathrm{P}$. Gaxotte, G. Duhamel, M. Garçon ne manquent pas de «monter au créneau » avec plus ou moins de nuances ${ }^{11}$. D'un côté comme de l'autre, l'argument selon lequel atteinte est portée à la langue française est souvent repris. Tout comme celui selon lequel on voudrait soit sacrifier la littérature française sur l'autel d'une efficacité statistique réductrice, soit priver les étrangers et les colonisés d'un accès aux grands textes de cette même littérature ${ }^{12}$, soit les deux à la fois.

La mise en cause politique et l'accusation d'atteinte à une langue d'écrivains se rejoignent d'ailleurs dans cette réaction vive d'Etiemble, qui n'est pas encore parti en croisade contre le " franglais ", mais qui, dans la revue Les Temps modernes, écrit en août 1952 un article intitulé "De la prose française au sabir atlantique", où l'on peut notamment lire :

Je sais, je sais: en même temps qu'ils détruisent notre langage, nos zélés protecteurs nous en offrent un "efficient ersatz" aussi basiquement bas que leur "Basic English". Que les Anglo-saxons aient du goût pour le volapuk, ça les regarde ; mais que notre ministère de l'Éducation nationale gaspille ses rares millions à confectionner notre ruine, cela nous regarde: et nous au tout premier chef, écrivains de la langue française; nous payons des impôts, de lourds impôts, mais non pas pour que cet argent serve à financer la mort de notre langue. C'est vrai, grâce au français basique, "li mon ami" li pali basique, li piti nègue itou, ya bon poulunion française" : et puis quand l'adjudant Smith donnera ses ordres au cher adjudant Flick, "loui palé bésic fuancé, toué facil, toué bon pour ponne vèremaquete, toué bon pour toué russes." Eh bien nous ne marchons pas. On veut bien se faire tuer, à la rigueur, pour que les survivants conservent le droit de parler notre français; qu'est-ce qu'une patrie qui n'a plus de langage? (Les Temps modernes, août 1952 : 303).

On ne s'attardera pas sur la véhémence du ton de pamphlétaire qui caractérise souvent les écrits d'Etiemble, mais on relèvera qu'il s'agit d'un texte paru dans Les Temps modernes, revue dont le directeur fondateur a été J. P. Sartre, et que l'attaque portée télescope, outre l'argument d'attentat contre la langue, une accusation de gabegie des crédits, un certain anti-américanisme (le sergent Smith atlantique), une forme d'antigermanisme (la bonne Wermacht) et une parodie du « petit nègre » tout juste bon pour l'Union française. En bref, on trouve ici en quelques lignes comme un condensé violent des multiples angles de tir contre le projet d'élaboration d'un Français élémentaire. 


\subsection{Un instrument de l'école capitaliste?}

débats autour de l'école. Il se trouve en effet de nouveau sur la sellette dans l'ouvrage publié en 1971 par C. Baudelot et R. Establet, L'école capitaliste en France. Le Français fondamental, où les critiques venant d'une tradition élitiste et conservatrice voyaient une menace de dégradation et quasiment de retour à la barbarie, apparaît à Baudelot et Establet comme un bastion, parmi d'autres, du pouvoir en place, comme un modèle linguistique et culturel qui contribue au renforcement et à la reproduction des hiérarchies sociales dans une école qui, pour la bourgeoisie, est une fabrique d'héritiers, et, pour les enfants de milieux socioculturels défavorisés, un labyrinthe aux innombrables culs de sac et aux sorties prévisibles et plus que limitées.

La charge contre ceux que Baudelot et Establet appellent «les linguistes de SaintCloud», repose moins sur le Français fondamental que sur des usages pédagogiques particuliers de ses résultats, notamment dans des méthodes de lecture destinées aux travailleurs immigrés. Elle porte largement à faux, fait feu de citations choisies auxquelles d'autres pourraient être opposées, mêle les publics et les enjeux ${ }^{13}$. Mais elle rejoint des questions de fond qui n'ont rien perdu de leur actualité, s'agissant de la langue de scolarisation. Au-delà des critiques touchant au contenu idéologique de tel ou tel instrument pédagogique, elle interroge, pour les enfants scolarisés, le principe même d'un contenu d'enseignement pré-limité et « imposé » :

Nous dirons en conclusion que le français correct imposé à l'école primaire non seulement ne parle pas d'eux aux enfants des classes populaires, mais il empêche ces derniers d'en parler, et cela de trois façons :

1. Il réprime aussitôt que formulé tout ce qui pourrait apparaître comme l'expression spontanée de leur monde et de leur vie...

2. Il oblige alors les enfants à garder leurs problèmes...pour eux...

3. Il ne leur donne aucun moyen de formuler en les exprimant verbalement ces conditions réelles : il leur fournit au contraire un substitut : le discours scolaire [...] La fonction de communication de la langue est ici sacrifiée au profit de la conformité de la norme: celle du français fondamental qui permet de lire $\mathrm{M}$. Genevois, A. Maurois, G. Duhamel, mais non un bulletin syndical. (L'École capitaliste en France : 231-232)

Il est plaisant de relever que le français fondamental permettrait ici la lecture de G. Duhamel, celui-même qui y voyait, vingt ans plus tôt, une sorte de français croupion! Mais cette citation manifeste surtout le glissement auquel procèdent Baudelot et Estabel, dans la mesure où les promoteurs du Français élémentairen'envisageaient aucunement que cet instrument détermine un contenu d'enseignement limité et imposé pour l'école élémentaire en France ${ }^{14}$. Elle manifeste aussi à quel point le français fondamental est rapidement devenu, autant qu'une référence durable, un lieu de mémoire.

Au long de quelques citations rassemblées ici, on aura peut-être perçu comment l'entreprise novatrice qu'a représentée l'élaboration du Français élémentaire s'inscrit dans un contexte d'évolution, non seulement de ce qu'on n'appelle pas encore alors les sciences du langages (avec les débats autour de la constitution de corpus et le recours à la statistique), mais aussi des représentations de la langue, de la communication et du français. Les enjeux idéologiques et politiques se concentrent notamment sur la défense de l'intégrité d'une langue de transmission culturelle et/ou d'émancipation sociale, 
dans cette période historique d'interrogation sur l'avenir de ce qui fut l'empire colonial et sur les relations entre atlantisme et communisme. On sent que, dans ces débats publics, l'oralité n'est plus ou n'est pas encore un objet central, et le corpus recueilli, peu discuté quant à sa constitution, n'est pas étudié sous cet angle. La dimension communicationnelle ne sera pleinement thématisée que plus tard. C'est d'abord en termes de conception du langage et de politique de la langue que s'ordonnent les positions en présence.

\section{BIBLIOGRAPHIE}

BESSE, H. (1979), « Contribution à l'histoire du français fondamental », Le Français dans le monde, 148, pp.33-30.

BAUDELOT, C. \& ESTABLET, R. (1971), L’Ecole capitaliste en France, Paris : François Maspéro.

CHEVALIER, J.-Cl. \& ENCREVÉ, P. (2006), Combat pour la linguistique, de Martinet à Kristeva, Lyon : ENS Éditions.

COHEN, M. et al. (1955), Français élémentaire, non! Paris : Éditions Sociales.

COSTE, D. (éd.) (1984), Aspects d'une politique de diffusion du français langue étrangère depuis 1945. Matériaux pour une histoire, Paris : Hatier.

COSTE, D. (1989), « Aurélien Sauvageot (1897-1988). Un aspect de son œuvre : aux origines de l'élaboration du Français fondamental », Études de Linguistique Appliquée 75, pp. 24-39.

GADET, F. (2008), « Le livre, le caméléon ou le singe savant », Documents SIHFLES 36.

GALAZZI, E. « 1950 : où est passé l'oralité ? Le français dans le monde, Recherches et applications, 43.

GOUGENHEIM, G. (1952, 1953, 1954), pages d'information sur l'avancement du projet, L'Éducation nationale, numéros des 17 janvier 1952, 7 mai 1953, 14 octobre 1954.

GOUGENHEIM, G. (1956), « Français élémentaire? Oui ou non? », Bulletin de la Faculté des Lettres de Strasbourg, 8-9.

GOUGENHEIM, G. (1962), « Principes nouveaux pour l'enseignement du français », Esprit 11, pp. 585-594.

GOUGENHEIM, G., MICHÉA, P., RIVENC, P. \& SAUVAGEOT, A. (1956), L'élaboration du Français élémentaire, Paris : Didier.

GENOUVRIER, E. \& PEYTARD, J. (1972), Linguistique et enseignement du français, Paris : Larousse. HESSEL, S. (1984), « Mutations », Aspects d'une politique de diffusion du français langue étrangère depuis 1945. Matériaux pour une histoire, Coste D. (éd), Paris : Hatier, pp. 50-52.

RIVENC, P. (1956), Réponse au compte-rendu fait de « Français élémentaire ? Non » par Roger G., Europe, 121-122.

RIVENC, P. (1973), « A l'aube de l'ère des corpus », Voix et Images du CREDIF 18, pp. 12-16. 
RIVENC, P. (1979), « Le français fondamental vingt-cinq ans après ", Le Français dans le monde 148, pp. 15-22.

\section{NOTES}

1. Dont on sait qu'il deviendra, quelques décennies plus tard, membre de l'Académie française, sans d'ailleurs cesser de contribuer occasionnellement au journal de ses débuts et rejoignant une institution que l'élaboration du Français élémentaire n'avait pas enthousiasmée outre mesure.

2. Rappelons ici que la désignation «Outre Mer» s'applique alors à l'empire colonial dans son ensemble et ne se réduit évidemment pas au DOM-TOM d'aujourd'hui.

3. Des anciens de l'ENS ont créé et animé la Mission Laïque Française au début du XXe siècle et c'est un autre ancien, M. Blancpain, qui préside, dans les années 50 et pendant plusieurs décennies, aux destinées de l'Alliance française. La petite histoire retiendra que Marc Blancpain ne se montra guère favorable à la diffusion du Français fondamental et aux activités du CREDIF, quand ce Centre de recherche et d'étude pour la diffusion du français, succédera, à la charnière des années 50 et 60 , avec des missions beaucoup plus diversifiées et étendues, au Centre d'étude du Français élémentaire.

4. Ce centre tient de la fausse fenêtre et ne disposera pas de moyens et d'appuis comparables à ceux de son homologue pour le français. Mais la symétrie et la parité de façade étaient "politiquement correctes".

5. Cette opposition entre vie et sciences humaines d'un côté, calcul et mathématiques de l'autre, chez l'autorité qu'est Dauzat, est encore symptomatique de positions qui seront bientôt battues en brèche. P. Guiraud publie en 1954 (année de la publication du fascicule du Français élémentaire) aux P.U.F., l'ouvrage Caractères statistiques du vocabulaire et, à Utrecht, sa Bibliographie de la statistique linguistique. B. Quemada s'engagera aussi, à Besançon, dans cette voie novatrice.

6. Sur ce point, la position de Cohen se situe à l'inverse de celle de Dauzat quant au rapport entre linguistes et pédagogues.

7. Voir à ce propos les témoignages J. Dubois et de B. Quemada dans Chevalier et Encrevé 2006 (pp. 156- $159 ; 213$ ).

8. L'article, publié en pleine page sur quatre colonnes, est intitulé, en grosses capitales, «UN FRANÇAIS ROBOT », avec en sous-titre : « Un article de Marcel COHEN sur le 'basic-french' ».

9. Pour les différents projets mentionnés par Marcel Cohen voir notamment Chevalier et Encrevé 2006 et, à propos de l'intérêt de Cohen pour l'oral, Galazzi (à paraître). Le projet d'Inventaire de la langue française, initié par Mario Roques, verra le jour sous une autre forme au début des années 1960, à Nancy, sous la houlette d'abord du recteur Paul Imbs, et aboutira à la constitution du Trésor de la Langue Française, le fameux TLF, aujourd'hui informatisé.

10. Gougenheim fait le choix de donner simplement, dans l'hebdomadaire officiel L'Education nationale, de brefs bilans d'étape des travaux en cours (Gougenheim 1952, 1953, 1954).

11. Cité par Les nouvelles littéraires du 11 janvier 1952, M. Garçon n'y va pas de main morte : « On ne va tout de même pas fabriquer un français petit-nègre pour étrangers fainéants ou incapables! Le français est une langue à laquelle il n'est pas question de rien toucher. » Ses pairs font généralement preuve de plus de mesure et tous les académiciens ne se montrent pas ouvertement hostiles à l'entreprise.

12. M. Cohen, dans son article des Lettres françaises du 7 février 1952 : «On veut faire mine de laisser au français, cette langue de si haute réputation, une place dans l'enseignement de pays variés. Mais on désire prendre toutes les précautions voulues pour se garder de la contagion de cette littérature où il $\mathrm{y}$ a tant de choses si dangereuses. » 
13. Elle présente en particulier une sorte d'amalgame abusif entre Le Français élémentaire et les propositions du Plan de rénovation de l'enseignement du français à l'école élémentaire, dit «Plan Rouchette ", objet de débats dans cette période post 1968.

14. Tout au plus, comme le rappellent ici même A. Bandelier et C. Cortier, des auteurs d'échelles pour l'enseignement de l'orthographe ont pu tirer parti, entre autres, des listes de mots du Français fondamental. Par ailleurs, en 1972, E. Genouvrier et J. Peytard, dans l'ouvrage Linguistique et enseignement $d u$ français, insistent sur le parti qui peut être tiré du Français fondamental, mais certainement pas comme d'un syllabus pour le français « langue maternelle ».

\section{INDEX}

Keywords : elementary French, languages representation, linguistic policy, French Language Expansion, stakes

Mots-clés : français élémentaire, représentation des langues, politique linguistique, diffusion de la langue française, enjeux

\section{AUTEUR}

\section{DANIEL COSTE}

École normale supérieurs Lettres et Sciences humaines 